\title{
The components of latency of response in two models for auditory detection with deadlines
}

\author{
RAY PIKE and LEN DALGLEISH \\ University of Queensland, St. Lucia, Queensland, Australia 4067
}

\begin{abstract}
Two models are described for the case of auditory detection with response time deadlines. These are the "timing" model, modified from Luce and Green (1972), and the "interval of uncertainty" (IU) model, modified from the "counting" model of McGill (1967). The two models are expressed in the form of additive component latencies, including a component which varies inversely with probability of response and a component for a longer response on the "nondeadline" response condition. An experiment is described, incorporating deadlines on both $s$ and $n$ trials separately, and the additive component latencies of the models are calculated. The "interval of uncertainty" model is seen to represent the data better than the "timing" model, even though different versions of the latter model are considered. Parameters are then fitted to give a representation of the IU model. The data from one subject of another study not employing deadlines can also be fitted to the model, and an interesting problem then arises concerning the exact locus of the speed-accuracy tradeoff. It is concluded that the IU model needs extending if it is to describe the data without the use of a component of latency which varies inversely with response probability.
\end{abstract}

A recent paper by Pike, McFarland, and Dalgleish (1974) compared two speed-accuracy tradeoff models for auditory detection when the observer is instructed to respond within a specified deadline time. The two models were those of Luce and Green (1972), based on a "timing" process, and of McGill (1967), based on a "counting" process. The conclusion reached by Pike et al. (1974) is strongly in favor of a modified counting model, but discrepancies existing between its predictions and the data make it necessary to consider the possibility of other response time components affecting the overall latencies. The general strategy of the present study is to reexamine the two models after these components have been accounted for.

A more complete description of both models is given in Pike et al. (1974). The timing model is essentially that used by Green and Luce (1973), with the simplification that only one timing channel is assumed to operate. This assumption does not, as far as can be determined, greatly affect predictions concerning mean latencies. The timing model considered here is thus a signal detection model with time (strictly, the average interarrival time, IAT, of a constant number of neural impulses) as the decision axis. It is important to note that an observation position is thus directly related to response time. The pulse rate varies with stimulus intensity, and the signal distribution has a shorter mean IAT than that of noise because of a faster associated "pulse" rate. Since the number of pulses counted is constant, an increase along the decision axis is associated with a

This study was supported by an award from the Australian Research Grants Committee. Requests for reprints should be sent to Ray Pike, Department of Psychology, University of Queensland, St. Lucia, Queensland, Australia 4067. decrease in neural pulse rate and hence an increasing likelihood of an $n$ trial. The stimulus distributions are, therefore, reversed in position to what they usually are in diagrams of the TSD model. The distributions are gamma in form, but here will be taken to approximate normal distributions (cf. Luce \& Green, 1972).

The McGill counting model is a signal detection model with discrete Poisson distributions (of "counts" of pulses per constant time interval). As modified by us, however, the model has two decision criteria. If an observation ("pulse" count) falls within the interval defined by the two criteria, it is ignored and a further observation is taken, until one occurs outside of the interval. The critical features of this model are all determined by this interval of uncertainty (IU), and we will henceforth refer only to the $I U$ model, which will be the usual signal detection model with normal distributtions of $n$ and $s+n$ but with an interval of no decision defined by two criteria, instead of the usual single criterion. In terms of the McGill model, the decision axis can represent "count" per unit time but, clearly, the model has a more general application. One important aspect of the model is that the interval width is assumed to be under observer control, varying with the speed-accuracy conditions of the experiment. The wider the interval, the more accurate the response but the larger the response time, due to the greater number of observations required, on the average, for a decision. Formulae for response probabilities and mean latencies of the model are derived in Pike et al. (1974) and are given briefly in Note 1 . The model considers multiple observations as occurring within a trial, where there is only one stimulus presentation. Previous considerations of an interval of uncertainty in signal detection 
have usually been related to repeated stimulus presentations (e.g., Swets \& Green, 1964), although a model of Sandusky (1971) considers a "band" criterion for two-category signal recognition situations and the sequential decision model of Laming (1968) may also be interpreted in terms of two criteria and multiple observations.

In the Pike et al. (1974) study, two deadline conditions were used. The s-deadline condition was one where, if observers considered the stimulus to be a signal (an $s$ trial), they were to respond before a deadline time. No deadline was imposed on $\mathbf{n}$ trials in this condition. The converse applied in the n-deadline condition. When these particular conditions are imposed, as they will be here, the count parameter of the timing model may be expected to change since it is fixed so that the deadline time is rarely exceeded (cf. Green \& Luce, 1973). This parameter should then be less in the $n$-deadline condition since it is assumed that the pulse rate is less on $\mathbf{n}$ trials. Consequently, if the timing process is in operation on the n-deadline condition, the reduction in the count parameter must decrease comparative accuracy because of the decrease in neural pulse sample size of the timing process, and hence the calculated value of $d$ ' should decrease from $\mathrm{s}$ to $\mathrm{n}$ deadline.

The two models differ markedly in predictions concerning mean latency when there is a bias towards "no," and the experiment to be reported here is, therefore, restricted to this condition. The following qualitative predictions may be made for the two models based on decision time only, when no consideration is given to residual response time components.

The predictions of the timing model with regard to mean latency are easily seen, since the decision axis in this model is directly related to response time and the $\mathbf{s}+\mathbf{n}$ distribution is below the $\mathbf{n}$ distribution. Consequently, it is the case (for normal distributions with equal variance at least) that the mean latencies for the four response categories may be ordered

$$
\mathrm{L}(\mathrm{H})<\mathrm{L}(\mathrm{FA})<\mathrm{L}(\mathrm{M})<\mathrm{L}(\mathrm{CR}),
$$

and further, that "yes" latencies are less than "no" mean latencies and that s-trial mean latencies are less than n-trial mean latencies. It would take gross differences in variance of the distributions to upset these predictions, at least for other than small values of $d^{\prime}$. A strategy we use is to take the mean values along the decision axis of the truncated distributions, one for each response category, and thus form an interval scaling of the mean latencies; this will be utilized in the specification of parameters reported later. Another latency feature of the timing model is that correct mean latencies are greater than incorrect mean latencies except for criterion positions beyond the mean of then distribution (see Pike et al., 1974), which will certainly not be the case in the experiment reported here.

The decision latency predictions of the IU model are, for a bias towards "no," quite different to those of the timing model. It is shown in Pike et al. (1974) that mean latencies for correct responses are less than, or equal to, those for incorrect responses. It is also shown in Note 1 that mean latencies for "yes" responses and "no" responses vary with the interval position such that we would predict that with a bias to "no," mean latencies of "yes" responses are larger. From the mean latency formulae, mean latencies for $\mathrm{s}$ trials are larger than those for $n$ trials when the interval is right of center.

The above features of the models apply only to the basic decision processes. In the Pike et al. (1974) study, it was generally found that the data corresponded with the features of the IU model. However, it should also be found for that model that hit and miss mean latencies are equal and correct-rejection and false-alarm mean latencies are equal, depending as they do directly on the interval areas. This feature was not found in the Pike et al. (1974) data, and it was concluded that this discrepancy with an "otherwise successful model could be explained either by the action of differential residual response latencies or by the fact that the observation sampling process is rather more complicated than is supposed in the simplest form of the IU model. In any case, the action of residual response components clearly needs to be added to the decision process to make a more complete comparison of the models, and the values of such components can then be subtracted from observed latency values to make comparison based on the above differential predictions of the two decision processes. The simple assumption of additivity of the components may, clearly, be questioned, but it appears to be a reasonable first step.

The components of response time we consider are: (i) an overall input and output time, based on sensory, encoding, response selection and motor components; (ii) the decision time, based on the latencies arising from either the timing process or the IU process; (iii) a residual component due to the deadline condition, such that there is an additional amount of latency included in "nondeadline" response; (iv) a component varying inversely with the probability of response.

These components are assumed to be additive and independent. The first component may be taken to be constant for any deadline condition, reflecting the operation of the basic input-output processes. The third component is specific to the single deadline situation. It is assumed that, on the average, the observer is likely to take more time when making a response if it has already been decided that it is a nondeadline response. Hence this component will 
occur in only two response categories for each deadline condition. The fourth component is to account for the fact that, in studies of latency in signal detection situations, it has been found that the longer latencies are associated with the smaller probabilities of response (e.g., Carterette, Friedman, \& Cosmides, 1965; Gescheider, Wright, Weber, Kirchner, \& Milligan, 1969; Pike \& Ryder, 1973; Sekuler, 1965). There are various explanations for this. One of these supposes that the latency of an observation varies inversely with its distance from the criterion (e.g., Gescheider et al., 1969). The inclusion of such an effect has a precedent in another model which uses the concept of a signal detection interval of no decision. Thus, Atkinson and Juola (1973) define such a function in connection with a "retrieval interval" for short-term memory processing. A second explanation for variation of latency with response probability is that a sequential decision process is responsible for the latencies of response so that multiple observations are also required outside of the interval (see Pike, 1973). Yet a third is an extension of the IU concept: it can be supposed that with each observation an amount is accumulated towards a response. This amount is zero within the interval and varies directly with distance from the interval, these amounts accumulating until a threshold level is achieved. Such a decision process would then be similar to the accumulator model of Vickers (1970).

Each of these three modifications would greatly complicate the model if an attempt was made to incorporate any of them in detail. Consequently, we make the simplifying assumption that this component of latency, whatever its cause, may be given as a simple linear function of response probability. This is as good an approximation as any in the circumstances.

With these latency components forming the overall reaction time, the two models may now be stated in terms of their component parameters. Different forms of the additive models have to be considered, however, because they differ in relationships between mean decision latencies of response categories.

The mean latency parameters for the IU model are:

Latency

Category s Deadline n Deadline

$$
\begin{array}{lll}
\text { Hit } & a_{s s}+b_{s}\left(1-P_{H}\right) & a_{s n}+b_{n}\left(1-P_{H}\right)+y \\
\text { FA } & a_{n s}+b_{s}\left(1-P_{F}\right) & a_{n n}+b_{n}\left(1-P_{F}\right)+y \\
\text { Miss } & a_{s s}+b_{s}\left(1-P_{M}\right)+n & a_{s n}+b_{n}\left(1-P_{M}\right) \\
\text { CR } & a_{n s}+b_{s}\left(1-P_{C}\right)+n & a_{n n}+b_{n}\left(1-P_{C}\right)
\end{array}
$$

where $a_{j k}$ denotes general input/output latency plus the IU decision process mean latency for trial stimulus condition $j$ (signal or no signal) on the $k$ deadline condition; $b_{\mathbf{s}}, b_{n}$ are the components accounting for the inverse relationship between probability and latency (we refer to this as the $b$ component); and $n, y$ are the extra latencies for responding "no" and "yes" on $s$ - and $n$-deadline conditions, respectively (we refer to this as the $y / n$ component).

The essential latency relationship in the above model is that the decision latencies for hit and miss categories and for FA and CR categories are equal. Evaluation of the parameters as indicated above ensures this condition. that

For the timing model, the essential relationship is

$$
\mathrm{L}(\mathrm{H})<\mathrm{L}(\mathrm{FA})<\mathrm{L}(\mathrm{M})<\mathrm{L}(\mathrm{CR}) .
$$

As previously explained, the four decision latencies may be interval scaled given the mean position of the truncated distributions corresponding to the response probabilities. The mean decision latency (in interval scale units) of any response category will, therefore, be given by the average position of the relevant distribution area. For example, the interval scale value of the mean decision latency for a hit response will be given by the integral from minus infinity to $c$, the criterion position, of the product of $z$, the time axis, and the normal density (assuming the approximation) divided by the associated probability. The model may then be written:

$$
\begin{array}{lll}
\begin{array}{l}
\text { Latency } \\
\text { Category }
\end{array} & \multicolumn{1}{c}{s \text { Deadline }} & \\
\text { Hit } & a_{s}+b_{s}\left(1-P_{H}\right) & a_{n}+b_{n}\left(1-P_{H}\right)+y \\
\text { FA } & a_{s}+d_{s}+b_{s}\left(1-P_{F}\right) & a_{n}+d_{n}+b_{n}\left(1-P_{F}\right)+y \\
\text { Miss } & a_{s}+i_{1} d_{s}+b_{s}\left(1-P_{M}\right)+n & a_{n}+i_{1} d_{n}+b_{n}\left(1-P_{M}\right) \\
\text { CR } & a_{s}+i_{2} d_{s}+b_{s}\left(1-P_{C}\right)+n & a_{n}+i_{2 n} d_{n}+b_{n}\left(1-P_{C}\right)
\end{array}
$$

where $a_{s}$, $a_{n}$ are measures of input/output plus baseline hit decision latency, $d_{s}, d_{n}$ are the differences between the decision latencies for hit and false alarms, $i_{k j}$ are the interval scale values calculated from the response probabilities by comparing interval scale latency values. The parameters $b_{s}, b_{n}, y, n$ are as for the IU model.

The present study aims to compare these two models in terms of their predictions, after removal of the estimated residual latencies. The values of the residual latency parameters $(b, y$, and $n)$ in the above additive models may be determined exactly (as in each case we have four equations in four unknowns) and the estimates based on them removed so that conclusions can be based upon the decision latencies alone.

The experimental task was confined to one of only one bias condition, that of a bias towards "no," which 
should produce a mean latency pattern difficulty to fit by both models. The use of a single bias condition is also advantageous because it was found (Pike et al., 1974) that varying bias for subjects in the deadline situation with weak signals produced an extremely difficult task for the average subject to perform.

\section{METHOD}

\begin{abstract}
Subjects
Eleven male and two female undergraduate students aged 18-30 served as subjects for the experiment. All subjects had had no previous experience in auditory or signal detection tasks and required extensive pretraining. Each subject participated in the experiment as part of course requirements and received up to a maximum bonus of 50 cents per session depending on his performance.
\end{abstract}

\begin{abstract}
Apparatus
The "signals" (1.000-Hz tones) were generated from a Levell-type transistor decade oscillator (Type TG-664). The signals were embedded in continuous wide-band white gaussian noise and incremented onto a noncontinuous carrier tone $(1,000 \mathrm{~Hz}$ at SPL $50 \mathrm{~dB}$ ). presented through TDH 39 binaural earphones.

The warning and stimulus marker lights were mounted on the response keyboard which also held the two (yes-no) response button switches. The presentation of $\mathbf{s}$ or $\mathrm{n}$ trials, carrier tone, white noise, and feedback to the subject on the accuracy of his response were automatically controlled. The feedback on deadline performance (exceeding or under deadline) was controlled by the experimenter. The subject was in an adjacent sound-treated room.
\end{abstract}

\section{Design and Procedure}

During the first training sessions, the SPL of the white noise and the signal increment were manipulated for each subject to produce a d' approximately equal to one. These stimulus levels were then kept the same for all sessions. The range of SPLs of the white noise for all the subjects was 64 to $70 \mathrm{~dB}$, and the signal increment was either 2 or $3 \mathrm{~dB}$ above the carrier tone. For "noise" trials, no increment occurred. The signal and no-signal trials were equiprobable.

The onset of the carrier tone was marked by a warning light which was followed $3 \mathrm{sec}$ later by a marked stimulus presentation where either the signal was or was not incremented onto the carrier tone. The signal (or no signal) and the carrier tone were response-terminated. The time between trials was constant at 9 sec.

The experiment contains two treatment conditions (signal deadline and no-signal deadline), and data were obtained for each subject on each of these conditions.

The order in which the treatment conditions were undertaken varied for the 13 subjects and was determined by using a balanced ABBA order. Seven subjects completed the s-deadline condition first and six subjects completed the n-deadline condition first. After two or three training sessions without deadlines, each subject completed 100 practice trials and then 300 experimental trials in each deadline condition. Experimental sessions were approximately $1 \mathrm{~h}$ in duration and contained about 200 trials. Each session consisted of three blocks of trials, and these were separated by a 4-5-min "rest" period for the subject. When the subject was changing from one deadline condition to another, the first 100 trials in that session were practice trials.

During the course of any experimental session, the subject was permitted one of two responses, "yes" (Y) or "no" (N) corresponding to the two types of trials. The response was made by pressing either index finger held on the response buttons, the yes/no buttons being alternated between subjects. The subject was instructed to respond as quickly and as accurately as possible. Following the response, the subject received feedback automatically with regard to the correctness of his decision and, if it was a deadline trial (either $\mathbf{s}$ or $\mathbf{n}$ depending on the condition), information was given on whether or not the response was made before the prescribed deadline which was set at $600 \mathrm{msec}$ and was kept constant throughout the experiment. (The subjects were instructed to maintain a bias towards saying "No" by use of the instruction, "If you are in doubt, respond No.")

To enforce deadline performance, subjects were fined 20 points if they exceeded the prescribed deadline. If the subject anticipated the stimulus by respond ing prior to the stimulus marker light, he was fined 25 points. These points were totaled for each experimental session and converted to a molietary bonus which was based on a possible 50 cents maximum for the session.

\section{Results and Discussion}

Our instructions to the subjects produced only small amounts of bias. Indeed, one of the 13 subjects displayed a bias to responding "Yes," and since it was essential to the comparison of the two models that a bias to "no" be obtained from each subject, the data of this subject were omitted from the analysis. For the other 12 subjects, the probabilities for the four categories of response and values of $d^{\prime}$ and $\beta$ based on overall frequencies are given in Table 1.

The component models have four parameters for each deadline condition, two of which are directly related to the decision process and two of which are "extra" time components. The latter are the b components and the $\mathrm{y} / \mathrm{n}$ components, and our strategy is to remove these components and then test the predictions of the models on the remaining adjusted mean latencies. It should be noted, however, that all contrasts are being tested against the overall latency error variance, which should result in underestimation of the $F$ ratios.

The first question to be asked is whether our assumption of the $y / n$ component is justified, and accordingly we test the interaction between the deadline factor and the yes/no factor by forming the appropriate interaction contrast defined on the eight mean latencies given in Table 1 . The interaction is in the predicted direction, and the comparison is significant at the $p<.01$ level $(F=9.43, d f=1,77)$. The $y / n$ component may now be subtracted from the mean latencies, as is done in Table 1 . It would appear from the adjusted means that the $b$ component may indeed exist, but the comparisons to test for this effect, those between correct and incorrect mean latencies, is not significant. However, notwithstanding the absence of statistical justification, it would still appear meaningful to include this component because, as previously mentioned, it reflects a feature which is generally found in TSD data.

Subtracting the b component, we obtain the a components (in/out time plus decision) for the two models, as given in Table 1 . Comparisons ${ }^{2}$ can now be made on the adjusted mean latencies, for each model, to test its basic predictions as indicated in the introduction (i.e.. IU model for latencies; $s>n$, yes $>$ no, incorrect $>$ correct; timing model; vice 
Table 1

Probabilities and Mean Latencies of Response in the Two Deadline Conditions and the Calculated Latency Components for the IU and Timing Models

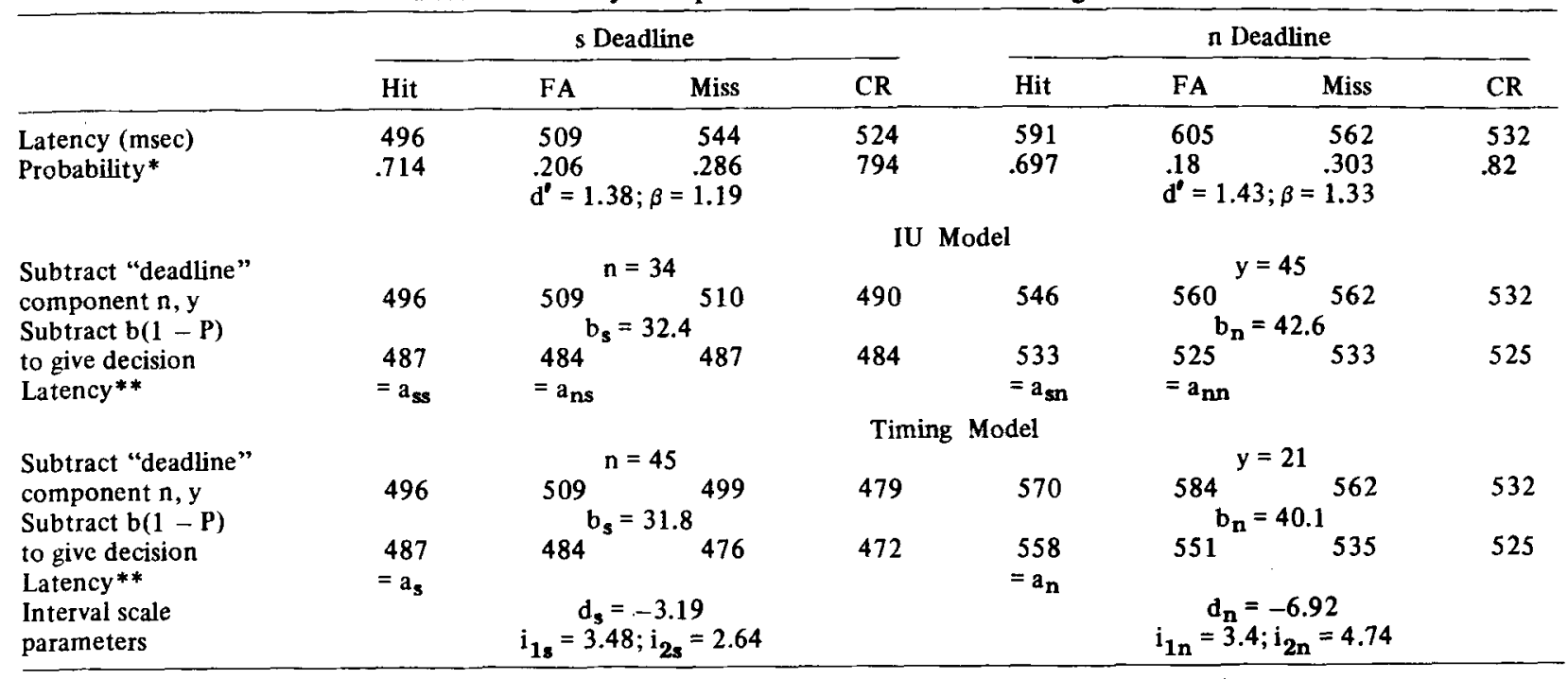

*Calculated on overall frequencies.

**Including input/output time.

Note-The b components, $n / y$ components, and $d_{s}$ and $d_{n}$ are in milliseconds.

versa). None of these comparisons were significant, which is not surprising considering the smallness of the difference between the relevant means (see Table 2). However, whereas all the trends in these tested differences are in accord with the IU model, there are none in the case of the timing model, where the trend in the mean latencies is completely opposite to that predicted. A factor which contributes to the lack of statistical significance in the IU model adjusted latencies is the fact that the obtained bias was only small and neutral bias in the model results in the prediction of no differences in the adjusted means. It suggests that if we can calculate the intervals upon the decision axis, they should be so positioned as to exhibit only a small amount of bias. It also suggests that we should examine data from other detection studies where there is more extreme bias to verify the model predictions. This we do later.

\section{Variations of the Timing Model}

In view of the poor performance of the timing model in matching the results, it was decided to consider alternative versions of its component structure. There are five modified versions we consider, and these are: Version 1 -Omits the $b$ component; parameters calculated by least squares. Version 2-Calculates $y / n$ component directly; $b_{1}$ for $s$ distribution, $b_{2}$ for $n$ distribution. Version $3-$ Calculates $y / n$ component directly; $b_{1}$ for "yes" responses, $b_{2}$ for "no" responses. Version 4-Omits $y / n$ component; $b_{1}$ for $s$ distribution, $b_{2}$ for $n$ distribution. Version 5-Omits $y / n$ component; $b_{1}$ for "yes" responses, $b_{2}$ for "no" responses.
Version 1 has only three parameters, which may, therefore, be estimated by least squares. In Versions 2 and 3 , the $y / n$ component is estimated simply by taking the overall difference between "yes" and "no" mean latencies for the separate deadline conditions. The allocation of the $b$ parameters in Versions 2 and 4 may allow for the possibility of unequal variances in the $s$ and $n$ distributions, whereas in Versions 3 and 5 it allows for a different function on each side of the criterion.

Table 3 gives the adjusted mean latencies for these five modifications of the timing model. It will be seen that Modifications 1 and 3 do not improve the situation. Modification 2 has produced the predicted trend in the mean latencies by virtue of a small positive $d$ value. As may be seen, however, the differences between these mean latencies are now very small. This poses a further problem, at least for the single channel timing model, as follows. The distributions of $s$ and $n$ are those of the time to a count or sum of $k$ "pulses," and their variances will,

Table 2

Mean Latencies for Categories of Response in the IU and Timing Model

\begin{tabular}{lllll}
\hline & \multicolumn{2}{c}{ IU Model } & \multicolumn{2}{c}{ Timing Model } \\
& s-d & n-d & s-d & n-d \\
\hline Yes & 486 & 531 & 487 & 557 \\
No & 485 & 527 & 473 & 530 \\
Signal & 487 & 533 & 484 & 551 \\
No Signal & 484 & 525 & 475 & 530 \\
Correct & 485 & 528 & 479 & 540 \\
Incorrect & 486 & 530 & 479 & 541 \\
\hline
\end{tabular}


Table 3

Calculated Components and Decision Latencies for Five Versions of the Timing Model

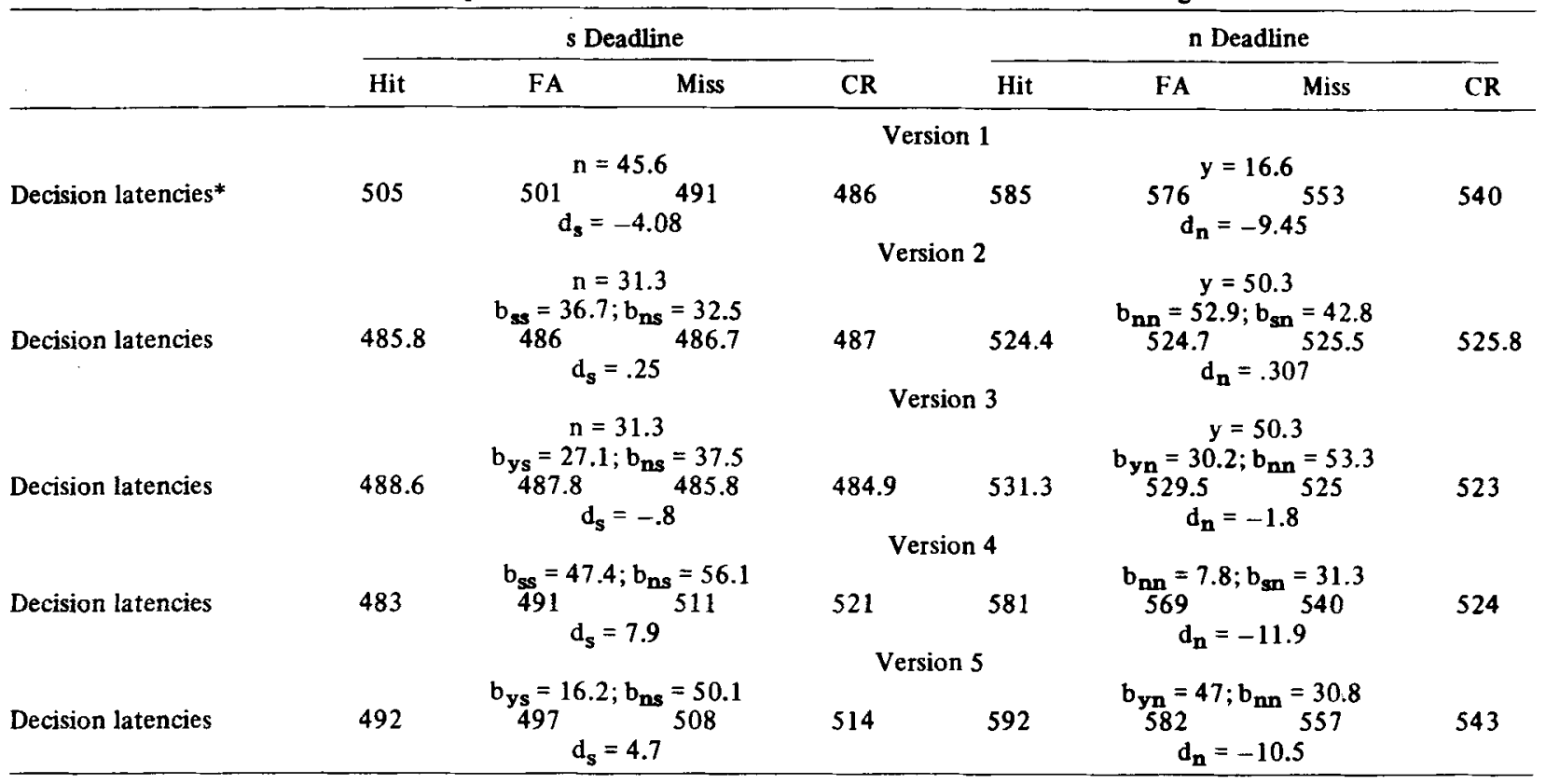

*Including input/output time.

therefore, be approximately $k$ units of variance (where one interarrival time has a unit variance and assuming IATs are independent; cf. Luce \& Green, 1972). Thus the ratio of the standard deviation (in milliseconds) of a detection distribution to the time from zero to the distribution mean is of the order of $\sqrt{k} / k$, or $1 / \sqrt{k}$. Now we can make a rough estimate of the standard deviation. based on the differences between the mean latencies, and consider the ratio of this to the decision time (say about $200 \mathrm{msec}$ at least). This ratio can then be equated to $1 / \sqrt{\mathrm{k}}$. It follows that $\mathrm{k}$ must be a very large number, say $200^{2}$, and this is a virtually impossible size of count for the single-channel model, and even for a multiple-channel model it would present considerable difficulty. Green and Luce (1973) have estimated the value of the product of $\mathbf{k}$ and the number of channels to be 50,32 , and 32 for three subjects, respectively, in an s-deadline situation with more powerful signals than those used here.

Versions 4 and 5 both permit the correct order of mean latencies for the s-deadline situation, and at more realistic intervals than those of Version 2. However, since the reverse order is present for $n$ deadlines, it is difficult to come to any firm conclusion. Possibly it may indicate the operation of a dual process (as has been suggested by Green and Luce, 1973, for the cases of sn deadline where equal mean latencies indicated the counting model, and $s$ deadline where larger $\mathbf{n}$ trial mean latencies indicated the timing model), so that the timing process operates in the s-deadline condition and the IU process in the $\mathrm{n}$-deadline condition (since the mean latencies for $\mathbf{n}$ deadlines could be explained by the IU process).
Clearly, however, this explanation can only be given serious consideration as a last resort, as it is very nonparsimonious. It is difficult to envisage a realistic decision system operating in this fashion.

A further result contrary to the timing model is that the obtained values of $d^{\prime}$ decrease from $n$ - to s-deadline conditions $(t=1.966, p<.05$, two-tail $)$. The IU model makes no explicit prediction concerning the relative $d^{\prime}$ values unless it is assumed that there may be a one-sided contraction of the interval in deadline situations such that this contraction is greater for the interval in the s-deadline situation if the overall bias is to "no" and in the n-deadline situation if the overall bias is to "yes." The present situation accords with the former case, in which case it predicts a larger $d^{\prime}$ for the n-deadline situation, the interval being less contracted. Although this appears here as a post hoc suggestion, a similar assumption was made in Pike et al. (1974) to account for differences in ROC curves.

In the present context, the IU model is clearly superior to the timing model. It may be thought that one way to improve the latter model would be to incorporate an interval of uncertainty for timing. Such a modification certainly increases the power of the model to explain patterns of mean latencies (see Pike et al., 1974), but there remains the predicted ordering of the mean latencies to make fitting of data difficult. For example, when there is a deadline on both stimulus conditions and a neutral bias, the mean latencies are equal for $\mathbf{s}$ and $n$ stimulus conditions, according to the study of Green and Luce (1973). This is easily explained by the IU model because there is 
now no $y / n$ component and because the interval is centrally positioned, with the result that decision latencies for $\mathbf{s}$ and $\mathbf{n}$ stimulus conditions are predicted to be equal. Green and Luce (1973) have concluded that the timing process may not operate in the sn-deadline situation.

\section{Representation of the IU Model}

The fact that the IU model can accommodate the present data better than the timing model can hardly be taken as a decisive test of the IU principle. The characteristics of the latency data which the model predicts could equally well be predicted by other sequential decision models (e.g., Pike, 1973). It is important, therefore, to ascertain if the model can be represented in a similar way to the usual TSD model. In the latter single-criterion model, response probability data from one experimental condition can always be represented by two distributions and the criterion. In the case of several bias conditions, however, some difficulty may arise in fitting the model-a problem related to that of estimating the best ROC curve (e.g., Abrahamson \& Levitt, 1969). We expect the same difficulties to arise but even more so in the IU model, since we are concerned with a more severe set of constraints. These constraints arise because (a) the interval must be the same width for both the $s$ and $n$ distributions of one deadline condition, and (b) various parameters should be constant across both deadline conditions. These constant parameters are $t_{0}$, the basic input/output time, $\mathbf{k}$, the time in milliseconds of one observations, and $\sigma$, the ratio of the standard deviation of the s-to-n distributions.

The parameters $t_{0}$ and $k$ are related to the three implicit probabilities of the $s$ distribution (i.e., the areas of the distribution partitioned by the two criteria) as follows. ${ }^{3}$

$$
\begin{aligned}
& \mathrm{p}_{\mathrm{s}}=\frac{\mathrm{k}}{\mathrm{a}_{\mathrm{s}}-\mathrm{t}_{\mathrm{o}}} \mathrm{P}_{\mathrm{s}} \\
& \mathrm{q}_{\mathrm{s}}=\frac{\mathrm{k}}{\mathrm{a}_{\mathrm{s}}-\mathrm{t}_{\mathrm{o}}} \mathrm{Q}_{\mathrm{s}} \\
& \mathrm{r}_{\mathrm{s}}=1-\left(\mathrm{p}_{\mathrm{s}}+\mathrm{q}_{s}\right)
\end{aligned}
$$

where $p_{s}, q_{s}, r_{s}$ are the implicit probabilities of "hit," "miss," and "uncertain" observations, respectively, and $P_{s}, Q_{s}$ are the observed hit and miss response probabilities. Similarly for the $n$ distribution.

Thus, given $t_{0}$ and $k$, the partition of each detection distribution can be determined. However, because of the constraint that each distribution interval must be the same width along the decision axis, a value of $o$ is required to fit the distributions simultaneously. Finding this value is an iterative procedure, since we are dealing with integrals of normal distributions.

Consequently, for one bias or deadline condition, there are many solutions-given the value of $t_{0}$ and $k$, there is a $\sigma$ which yields a solution. For two deadline conditions, the values of $a$ should be the same, for it is assumed that only the position of the interval changes. This constrains the solution and we are now able, apparently, to fix the value of $t_{0}$ and obtain a solution for $k$ and $o$. In this case, there is a conjoint range of values of the parameters which can give a representation of the model. For three bias conditions, the constraints imposed by keeping these three parameters constant are severe, and a complicated minimization problem may have to be solved to obtain the best representation. The use of variances to form additional equations is not feasible because we only have the variances for the total latencies and we require the variance of the decision-time component.

For the case of two deadline conditions, the iterative procedure we use is based upon the fact that the interval widths must be equal for each distribution within a condition. We minimize the sums of squares of differences between these within-condition widths to obtain the solution values of $k$ and $\sigma$. The value of $t_{0}$ we take is $175 \mathrm{msec}$ and is based upon the values found in the Green and Luce (1973) study, these being 165,168 , and $173 \mathrm{msec}$, for three subjects, estimated from their response times to high-intensity signals. The value of $\mathrm{k}$ is then found to be $259 \mathrm{msec}$, and $\sigma$ is 1.09 .

The representation of the IU model for our data is then as in Figure 1. As may be deduced from the mean latency results, given in Table 2 , the intervals are only slightly removed from the central position. The fact that the $n$-deadline interval is larger than the s-deadline interval indicates that the slight bias to "no" may, however, have a differential effect on the interval widths. Clearly, bias becomes a more complicated process in a model with two criteria.

The reason we require to illustrate the two deadline conditions separately is that the implicit $\mathrm{d}^{\prime}$ values, calculated for each condition, are different. They are 1.2 for the $s$ deadline and 1.124 for the $n$ deadline.

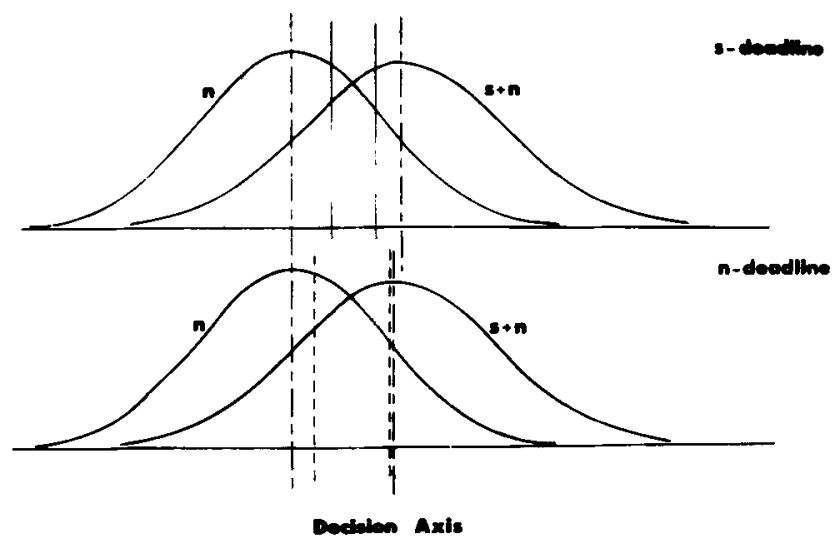

Figure 1. Representation of the IU model for s- and n-deadline conditions. 
Table 4

Mean Latency Data for Subject 1, Low-Intensity Signal Condition of Carterette et al. (1965) Together With Calculated Decision Latencies

\begin{tabular}{|c|c|c|c|c|c|c|c|c|}
\hline & \multicolumn{8}{|c|}{ Signal Presentation Probability } \\
\hline & \multicolumn{4}{|c|}{.2} & \multicolumn{4}{|c|}{.8} \\
\hline & Hit & FA & Miss & CR & Hit & FA & Miss & CR \\
\hline Latency (msec) & 1652 & 1543 & 1382 & 1357 & 1329 & 1455 & 1955 & 1969 \\
\hline Probability & .13 & .08 & .87 & .92 & .894 & .85 & .106 & .15 \\
\hline Subtract $b(1-P)$ to give decision & \multicolumn{4}{|c|}{$\begin{array}{c}\mathrm{d}^{\prime}=.279 ; \beta=1.42 \\
\mathrm{~b}_{2}=284\end{array}$} & \multicolumn{4}{|c|}{$\begin{array}{c}\mathrm{d}^{\prime}=.212 ; \beta=.788 \\
\mathrm{~b}_{0}=772\end{array}$} \\
\hline Latency & 1374 & 1307 & 1374 & 1307 & 1256 & $1326^{\circ}$ & 1256 & 1326 \\
\hline
\end{tabular}

The ratio of the values for $d^{\prime}$ becomes slightly smaller as $t_{0}$ decreases, but the value of $t_{0}$ then becomes unrealistic.

The general picture with regard to the interval position as depicted in Figure 1 does not change with other values of $k$ and $t_{0}$, but their width varies directly with $t_{0}$. The values of $k$ and $\sigma$ vary inversely with the value of $t_{0}$.

Clearly, then, the model is capable of producing a satisfactory representation, but there may be some difficulty in determining which representation is best. At present, solutions are somewhat limited insofar as the intervals are both in approximately the same position, as they must be in our situation, and they are both fairly central. It would be of some interest, therefore, to attempt to fit the model to two-condition data where the intervals are differently positioned and exhibit considerable bias. Also, we would like to see how the model may be applied to the nondeadline detection situation, for it clearly has this general application-indeed, it can be supposed that the notion of a double criterion may be interpreted as a general speed-accuracy tradeoff mechanism in any situation where detection, in the general sense, is involved. To further explore the representation of the model, we take the data from the auditory detection study of Carterette et al. (1965), Subject 1, lower intensity signal, with extreme bias conditions and submit it to the same procedure for estimating the additive component as outlined above, except that without the $\mathrm{y} / \mathrm{n}$ component (there were no deadlines) we solve for the $b$ components using least squares. Table 4 gives the additive component values for these data.

The range of solution values for the parameters $t_{0}$ and $\mathbf{k}$ for the Carterette et al. data is quite different from the range of values for our own data. The choice of parameter values is clear in this case, for at one point the implicit $\mathrm{d}^{\prime}$ values are equal. At this point, the value of $t_{0}$ is $415 \mathrm{msec}, k$ is $508 \mathrm{msec}$, and $\sigma$ is 1.03. This is a satisfactory representation of considerable interest, since the larger values required for $t_{o}$ and $k$ contrast with those or our own data and require some explanation. It should first be noted that the Carterette et al. study was of a different detection situation, with subjects not being aware that their responses were timed. As a consequence, it is possible that the stimulus/response encoding components of response time would have been very much larger than those from a deadline situation, so that the value of $t_{0}$ is considerably increased. It is possible to choose a value of $t_{0}$ smaller than the one indicated, but then the value of $k$ becomes larger and the implicit $d^{\prime}$ values depart from equality. The solution, as given, thus appears to be a fair compromise on the $t_{o}$ and $k$ values, and the $d^{\prime}$ values give further justification. However, the value of $k$ also requires explanationfor why should the observation time be longer in the nondeadline situation if, as we have suggested, the interval and, thus, the number of observations serve as a speed-accuracy tradeoff mechanism. Although the intervals for the Carterette et al. data, as depicted in Figure 2, are considerably wider than those from our data, this only partially accounts for the larger mean latencies.

The larger values of $\mathbf{k}$ are, we suggest, indicative that a locus of speed-accuracy tradeoff exists within each observation. For example, we have supposed that "counting," in the McGill (1967) sense, is the process determining each observation, so that each observation is the result of a count of neural "pulses". occurring in a constant time unit. Then, clearly, there is scope for a tradeoff mechanism; the longer the time allowed for the count, the more accurate the decision based upon it. We suggest, therefore, that in the Carterette et al. situation the observers are using a

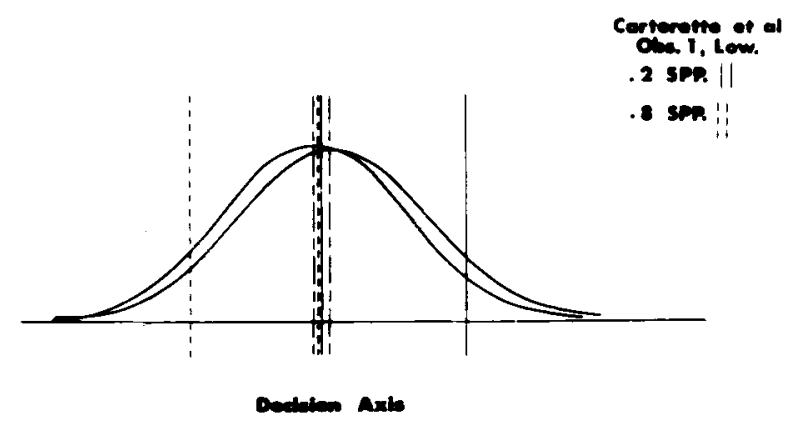

Figure 2. Representation of the IU model for the data of Carterette et al. (1965) Subject 1, low-intensity condition. Both intervals are in the same figure, the $d^{\prime}$ values being identical at a value of .16. 
much longer count time than they would in the deadline situation. It appears that such a tradeoff process may be the dominant one in the complete change from one experimental situation to another, whereas interval variation could be responsible for tradeoff in other cases. The possibility exists of an interaction between these tradeoff mechanisms, but this problem is one for future research.

The main weakness of the IU model, as it has been described here, is the necessity for the additive b component. This component is only a crude approximation, and its use will certainly lead to trouble with some data. For example, the data of the above Carterette et al. subject, for the higher signal intensity condition, are not so nicely represented, due to difficulties with the form of the $b$ component. The answer to this problem lies in showing that a more general IU model, as briefly described earlier in this paper, can account for the overall latency-probability relationship without the use of an extra component. Our efforts are presently directed towards this end.

\section{REFERENCES}

Abrahamson, I. G., \& Levitt, H. Statistical analysis of data from experiments in human signal detection. Journal of Mathematical Psychology, 1969. 6, 391-417.

Atkinson, R. C., \& Juola, J. F. Factors influencing speed and accuracy of word recognition. In $S$. Kornblum (Ed.). Attention and performance (Vol. IV). New York: Academic Press. 1973.

Carterette. E. C., Friedman, M. P., \& Cosmides, R. Reaction time distributions in the detection of weak signals in noise. Journal of the Acoustical Society of A merica, 1965, 38, 531-542.

Gescheider, G. A., Wright, J. H., Weber, B. J., Kirchner, B. M., \& Milligan. E. A. Reaction time as a function of the intensity and probability of occurrence of vibrotactile signals. Perception \& Psychophysics, 1969, 5, 18-20.

Green, D. M., \& LuCE. R. D. Speed-accuracy trade-off in auditory detection. In S. Kornblum (Ed.), Attention and performance (Vol. IV). New York: Academic Press, 1973.

Laming, D. R. Information-theory of choice reaction times. London: Academic Press, 1968.

LUCE, R. D., \& GreEN, D. M. A neural timing theory for response times and the psychophysics of intensity. Psychological Review, 1972, 79, 14-57.

McGrLL, W. I. Neural counting mechanisms and energy detection in audition. Journal of Mathematical Psychology, 1967, 4, 351-376.

Pirk, A. R. Response latency models for signal detection. Psychological Review, 1973, 80, 53-68.

Pire, A. R., McFariand, K., \& Dalgleish, L. Speedaccuracy tradeoff models for auditory detection with deadlines. Acta Psychologica, 1974, 38, 379.399.

Pixg, A. R., \& Rrdsr, P. Response latencies in the yes/no detection task: An assessment of two basic models. Perception \& Psychophysics, 1973, 13, 224-232.

SANdusky, A. Signal recognition models compared for random and Markov presentation sequences. Perception \& Psychophysics. 1971, 10, 339-346.

SexulrR, R. W. Signal detection, choice response times, and visual backward masking. Canadian Journal of Psychology, $1965,19,118-132$.

Swets, J. A., \& Greks, D. M. Sequential observations by human observers of signal in noise. In J. A. Swets (Ed.), Signal detection and recognition by human observers. New York: Wiley, 1964.

Vickers, D. Evidence for an accumulator model of psychophysical discrimination. Ergonomics, 1970, 13, 37.58.

\section{NOTES}

1. Define the distribution areas outside of interval as $p_{s}$ (implicit "hit") and $q_{3}$ (implicit "miss" 0 for the s distribution, and $p_{n}$ (implicit FA) and $q_{n}$ (implicit $C R$ ) for the $n$ distribution.

Then the corresponding response probabilities are:

$$
\begin{aligned}
& P_{s}=\frac{p_{s}}{p_{s}+q_{s}} ; Q_{s}=\frac{q_{s}}{p_{s}+q_{s}} \\
& P_{n}=\frac{p_{n}}{p_{n}+q_{n}} ; Q_{n}=\frac{q_{n}}{p_{n}+q_{n}}
\end{aligned}
$$

where $P_{s}=$ Prob. (hit) etc.

The corresponding theoretical mean latencies are then:

$$
L_{s}=\frac{1}{p_{s}+q_{s}} \quad \text { and } \quad L_{n}=\frac{1}{p_{n}+q_{n}}
$$

The expression for the "yes" mean latency minus the "no" mean latency is

$$
\frac{P(H) L(H)+P(F) L(F)}{P(H)+L(F)}-\frac{P(C) L(C)+P(M) L(M)}{P(C)+P(M)} .
$$

This reduces, after algebra, to an expression which is always positive except that it contains the term $\left(U_{3}-U_{n}\right)$ where $\mathrm{U}_{s}, \mathrm{U}_{\mathrm{n}}$ are the interval areas for $\mathrm{s}$ and $\mathrm{n}$ distributions. Clearly, this term is positive when the interval is to the right of center (bias to "no") and negative when the interval is to the left (bias to "yes"), assuming distributions of equal variance.

2. These comparisons will have coefficients formed from the response probabilities, the latter as given in Table 1 . As an example, the correct vs. incorrect comparisons for the IU model is in the form:

$$
\begin{aligned}
& \text { Prob(Hit)a } a_{s}+P_{\text {Prob(CR)a }} \text { Prob(Miss) } a_{s}+\operatorname{Prob}(F A) a_{n} \\
& \text { Prob(Hit)+Prob(CR) }-\overline{\text { Prob(Miss) }+ \text { Prob(FA) }}
\end{aligned}
$$

\section{Since}

$$
\begin{aligned}
& P_{s}=\frac{p_{s}}{p_{s}+q_{s}} \\
& P_{s}=\frac{p_{s}\left(a_{s}-t_{o}\right)}{k},
\end{aligned}
$$

$a_{3}-t_{0}$ being the decision latency and $k$ the time of each observation, both in milliseconds.

Hence,

$$
p_{s}=\frac{k}{a_{s}-t_{0}} P_{s}
$$

(Received for publication September 23, 1974; revision accepted November 3,1975 .) 